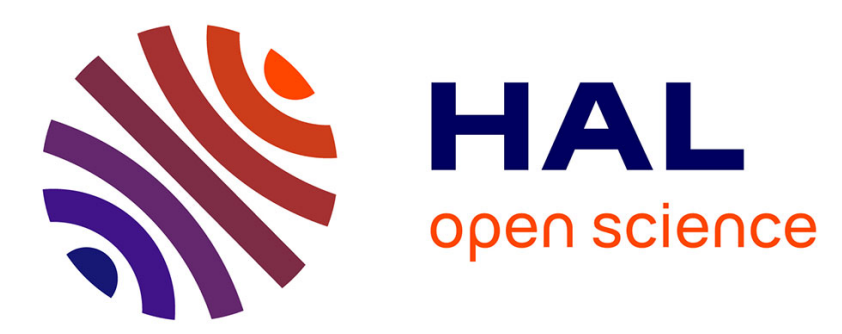

\title{
Multi-material additive and subtractive prosumer digital fabrication with a free and open-source convertible delta RepRap 3-D printer
}

Gerald C Anzalone, Bas Wijnen, Joshua Pearce

\section{- To cite this version:}

Gerald C Anzalone, Bas Wijnen, Joshua Pearce. Multi-material additive and subtractive prosumer digital fabrication with a free and open-source convertible delta RepRap 3-D printer. Rapid Prototyping Journal, 2015, 21 (5), pp.506-519. 10.1108/RPJ-09-2014-0113 hal-02119322

\section{HAL Id: hal-02119322 \\ https://hal.science/hal-02119322}

Submitted on 3 May 2019

HAL is a multi-disciplinary open access archive for the deposit and dissemination of scientific research documents, whether they are published or not. The documents may come from teaching and research institutions in France or abroad, or from public or private research centers.
L'archive ouverte pluridisciplinaire HAL, est destinée au dépôt et à la diffusion de documents scientifiques de niveau recherche, publiés ou non, émanant des établissements d'enseignement et de recherche français ou étrangers, des laboratoires publics ou privés. 
Gerald Anzalone, Bas Wijnen, Joshua M. Pearce, (2015) "Multi-material additive and subtractive prosumer digital fabrication with a free and open-source convertible delta RepRap 3-D printer", Rapid Prototyping Journal, 21(5), pp.506 - 519. doi:http://dx.doi.org/10.1108/RPJ-09-2014-0113

\section{Multi-Material Additive and Subtractive Prosumer Digital Fabrication with a Free and Open-source Convertible Delta RepRap 3-D Printer \\ Gerald C. Anzalone ${ }^{1}$, Bas Wijnen ${ }^{1}$ and Joshua M. Pearce ${ }^{1,2}$}

${ }^{1}$ Department of Materials Science \& Engineering, Michigan Technological University, Houghton, MI 49931, USA

2 Department of Electrical \& Computer Engineering, Michigan Technological University, Houghton, MI 49931, USA

\section{Structured Abstract:}

\section{Purpose}

Open-source RepRap (self replicating rapid prototyper) 3-D printers have made distributed manufacturing and prototyping an affordable reality. This paper presents novel modifications to a RepRap design that increase RepRap capabilities well beyond just fused filament fabrication as it is convertible between moving and fixed tool operation.

\section{Design/methodology/approach}

The design is a significantly modified derivative of the Rostock delta-style RepRap 3-D printer. Modifications were made that permit easy and rapid repurposing of the platform for milling, paste extrusion and several other applications. All of the designs are open-source and freely available.

\section{Findings}

In addition to producing fused filament parts, the platform successfully produced milled printed circuit boards, milled plastic objects, objects made with paste extrudates such as silicone, food stuffs and ceramics, pen plotted works and cut vinyl products. The multi-purpose tool saved $90-97 \%$ of the capital costs of functionally equivalent dedicated tools.

\section{Research limitations/implications}

While the platform was used primarily for production of hobby and consumer goods, research implications are significant since the tool is so versatile and the fact that the designs are open-source and eminently available for modification for more purpose-specific applications.

\section{Practical implications}

The platform vastly broadens capabilities of a RepRap machine at an extraordinarily low price, expanding the potential for distributed manufacturing and prototyping of items that heretofore required large financial investments.

\section{Originality/value}

The unique combination of relatively simple modifications to an existing platform have produced a machine having capabilities far exceeding that of any single commercial product. The platform provides users the ability to work with a wide variety of materials and fabrication methods at a price of less than $\$ 1,000$ providing users are willing to build the machine themselves.

Keywords: 3-D printing, additive manufacturing, distributed manufacturing, materials selection, metal processing, opensource, open-source electronics, open-source hardware, personal fabrication, RepRap

Article Classification: Technical Paper 
Gerald Anzalone, Bas Wijnen, Joshua M. Pearce, (2015) "Multi-material additive and subtractive prosumer digital fabrication with a free and open-source convertible delta RepRap 3-D printer", Rapid Prototyping Journal, 21(5), pp.506 - 519. doi:http://dx.doi.org/10.1108/RPJ-09-2014-0113

\section{Introduction}

RepRap (a portmanteau of self Replicating Rapid prototyper) 3-D printers were introduced as an open source technology (Jones, et al., 2011), essentially freeing 3-D printing from the frequently innovation-stifling effects of intellectual property law (Heller \& Eisenberg, 1998; Takalo \& Kanniainen, 2000; Boldrin \& Levine, 2002; 2008; Pearce, 2013; Jaffe \& Lerner, 2011; Chu, Cozzi \& Galli, 2012). RepRap designs are freely available and open-source, following the increasingly popular free and open-source software paradigm (Lakhani \& Von Hippel, 2003). The distinguishing feature of RepRap 3-D printers is that they are purpose-designed to employ as many printed components in their assembly as is possible (Sells et al., 2010). Structural components, gears, pulleys and any other part, be it high value or trivial, that can be printed by the printer itself are integral features of the designs, yielding the self replicating portion of the RepRap philosophy. RepRaps are acknowledged to have exceptionally high value (Wittbrodt, et al., 2013) as a result of this self-replicating feature and the fact that the designs are open-source and freely available. Since their introduction, RepRaps have been used for personal fabrication (Stemp-Morlock, 2010; Betts, 2010; Wittbrodt, et al., 2013), project-based education (Kentzer, et al., 2011; Irwin et al., 2014), the development of scientific tools, scientific visualization and experimentation (Partridge, Conlisk \& Davies, 2012; Pearce, 2012; Anzalone, Glover, \& Pearce, 2013; Zhang, et al., 2013; Pearce, 2014), and production of open-source appropriate technology for sustainable development (Pearce, et al., 2010).

There are presently two common RepRap printer designs; the Cartesian design having linear actuators parallel to each of three orthogonal planes, and the delta design (Rocholl, 2012) having three linear actuators arranged vertically around a circle. The Cartesian design is presently the most common of the two with many commercial products based upon it. Computer Numerical Control (CNC) and inexpensive microcontrollers (specifically the open-source Arduino and derivatives) are technologies that have made the printers possible. The ability to automatically and precisely control movement of the print head is what makes the platform a compelling candidate for repurposing. RepRaps typically print in polylactic acid (PLA) or acrylonitrile butadiene styrene (ABS). The recent development of the recyclebot, which is an open-source waste plastic extruder (Baechler, DeVuono, \& Pearce, 2013), and the commercialization of the entry-level extruders of similar design, not only showed a route to more environmentally friendly 3-D printing materials (Kreiger \& Pearce, 2013; Kreiger, et al., 2014), but also to expanded materials selection and composites. There is commercial RepRapcompatible filament available made from: nylon, polycarbonate (PC), polyvinyl alcohol (PVA), high impact polystyrene (HIPS), high-density polyethylene (HDPE), polyether ether ketone (PEEK), polyphenylsulfone (PPSF or PPSU), polyetherimide (PEI), polyoxymethylene (POM) and a number of polymer composites. In addition, since its inception, the RepRap community has been actively engaged in exploring possibilities beyond just printing polymer-based parts. Milling of printed circuit boards and experimentation with paste-based printing media have been underway on the RepRap wiki (http://reprap.org/) for years and many conventional printers have been retrofitted with apparatus that permit these capabilities.

As Cartesian 3-D printer designs are more common, most of the modifications have been based upon that platform. In nearly all Cartesian designs, the print head is one of the moving components and it moves parallel to the print bed in a simple $x-y$ axis for each layer. Since the hot end of the extruder is typically low mass, machine designs are typically not required to be exceptionally rigid. This presents a problem when retrofitting relatively heavy tools to the moving print carriage. Movement precision can degrade as a result of the additional mass. Insufficient frame rigidity manifests itself as uncontrolled movement during rapid changes in direction and the increased force required to accelerate the tool can cause motors to stall or slip. It also tends to be difficult to interchange tools with this platform. Thus, the ability to print with a wider selection of materials is limited because of the difficulty in switching heads - RepRap printers are optimized normally for one type of material printing.

A recent innovation to the delta printer design turns the printer upside-down such that the tool is fixed in place and the workpiece moves below it (Anzalone, et al., 2013; Haselhuhn, et al., 2014), which presents a solution: A convertible platform that is able to switch between a moving tool mode (e.g. conventional RepRaps) and a fixed tool mode.

The objective of this paper is to investigate a new a convertible RepRap platform capable of both moving and fixed tool operation. The bill of materials, basic assembly and description are provided as free and open source hardware. As the tool 
Gerald Anzalone, Bas Wijnen, Joshua M. Pearce, (2015) "Multi-material additive and subtractive prosumer digital fabrication with a free and open-source convertible delta RepRap 3-D printer", Rapid Prototyping Journal, 21(5), pp.506 - 519. doi:http://dx.doi.org/10.1108/RPJ-09-2014-0113

remains stationary in the stage configuration, a tool having much greater mass can be fit above the build platform, expanding the catalog of tools and thus materials available for both additive and subtractive manufacturing on the delta platform. The convertible delta RepRap is then tested with a variety of tools including: a thermoplastic extruder, a caulk/silicone extruder, a syringe pump extruder, a micro mill spindle and a tool holder used for vinyl cutting and plotting. Performance and capital costs are compared against single material commercial systems and the results are discussed. Future work is outlined for further enhancing both RepRap applications and materials selection in the burgeoning prosumer and maker communities.

\section{Materials and Methods}

The MOST (Michigan Tech Open Sustainability Technology Lab) Delta 3-D printer (MOST, 2014) was modified with replacement carriages having magnetic mounts facing both upwards and downwards and the closed top of the printer was replaced with an open ring. These changes permit conversion of the printer from its normal polymer-based printing configuration, in which the end effector with a hot end moves above a fixed build platform, into a 3-axis stage having the workpiece fixed to the end effector. One of the vertical boards on the printer was then replaced by a rigid aluminum rectangular tube to which a magnetic tool mount was attached, permitting easy and rapid swapping of tools positioned above the end effector. The magnetic mount, the design of the 3-D printed housing of which is shown in Figure 1a and 1b, consists of three high strength ring magnets encased in a 3-D printed housing attached to the fixed tool mount on the printer. The magnets are rated to hold $1.6 \mathrm{~kg}$. The magnets are held stationary by the 3-D printed casing and used to affix the tools. The surfaces of the magnets for the stationary tools are mated directly with the holder magnets.

As seen in Figure 2, the surface of the magnets on the end effector that attach to the steel balls of tie rods are lubricated with white lithium grease. To disengage a tool head it is manually removed from the end of the tie rods. A tool head can then be immediately manually placed in the same location if the orientation (end effector down or end effector up remains the same). If switching between down/up orientation the tie rods are completely removed from the system manually and moved in the opposite position (end effector down or end effector up). Then the new tool head/substrate holder is positioned on them.
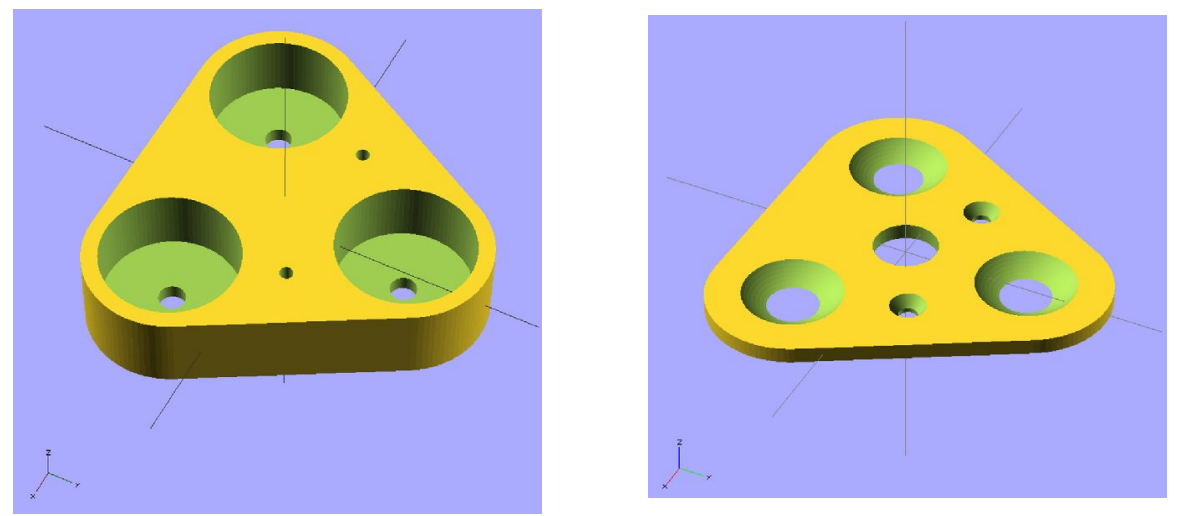

Figure 1. a) OpenSCAD rendered image of fixed tool magnetic mount base and b) top of base.

Tools are affixed to the magnetic mount by steel ball bearings that are integrated into the 3-D printed tool or tool holder. The bills of materials for conversion of a MOST Delta RepRap to a convertible RepRap is shown in Table 1 and for the magnetic base in Table 2. 
Gerald Anzalone, Bas Wijnen, Joshua M. Pearce, (2015) "Multi-material additive and subtractive prosumer digital fabrication with a free and open-source convertible delta RepRap 3-D printer", Rapid Prototyping Journal, 21(5), pp.506 - 519. doi:http://dx.doi.org/10.1108/RPJ-09-2014-0113

Table 1. Bill of materials to convert a standard MOST Delta RepRap to a convertible 3D printer/stage

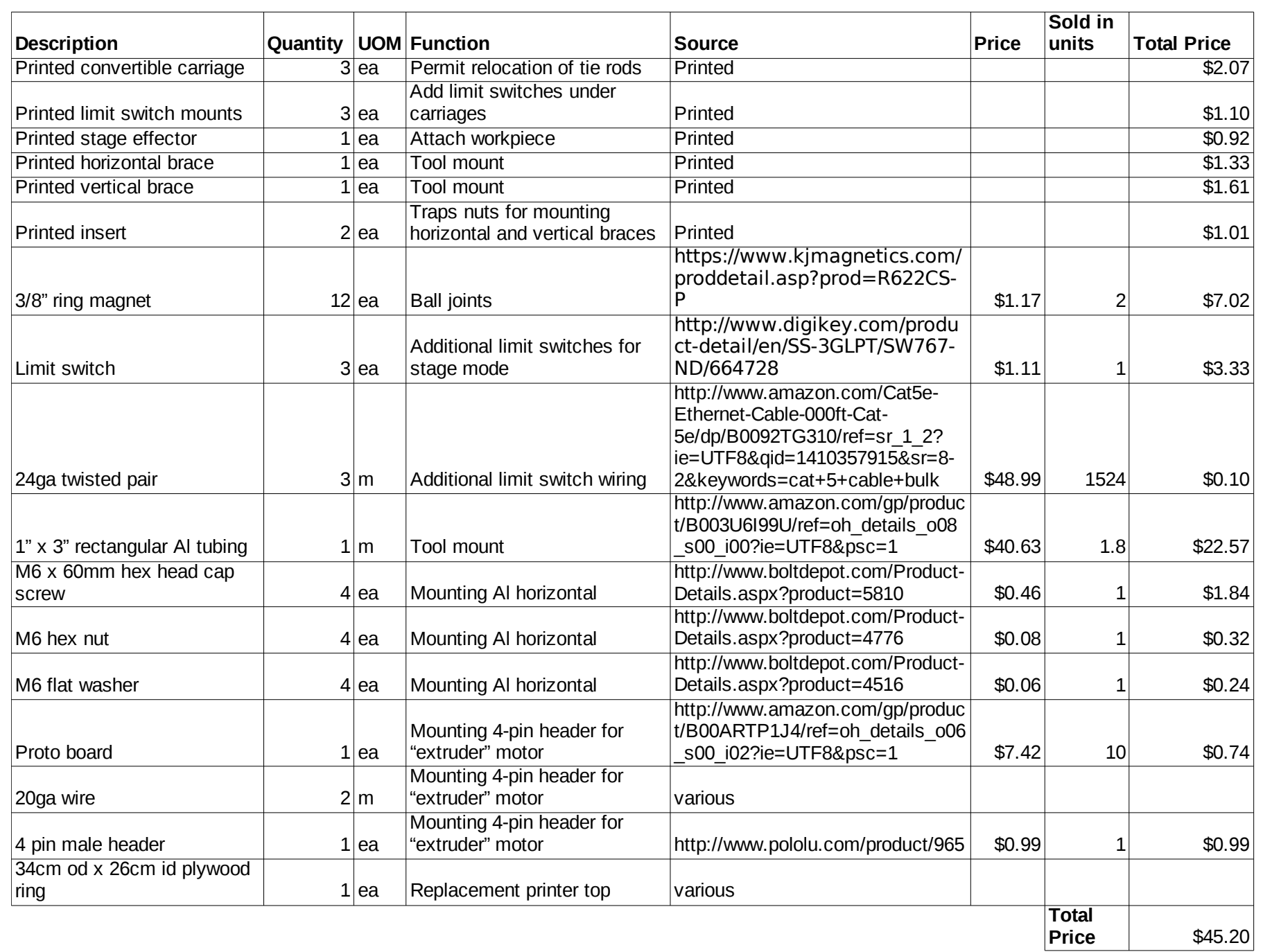


Gerald Anzalone, Bas Wijnen, Joshua M. Pearce, (2015) "Multi-material additive and subtractive prosumer digital fabrication with a free and open-source convertible delta RepRap 3-D printer", Rapid Prototyping Journal, 21(5), pp.506 - 519. doi:http://dx.doi.org/10.1108/RPJ-09-2014-0113

Table 2. Bill of materials for fixed tool magnetic mount

\begin{tabular}{|c|c|c|c|c|c|c|c|}
\hline Description & Quantity & UOM & Function & Source & Price & $\begin{array}{l}\text { Sold in } \\
\text { units }\end{array}$ & Total Price \\
\hline Printed cage top & 1 & ea & $\begin{array}{l}\text { Protect } \\
\text { magnets }\end{array}$ & Printed & & & $\$ 0.78$ \\
\hline 1" diameter x $1 / 2$ " ring magnet & 6 & ea & Hold tool & $\begin{array}{l}\text { https://www.kjmagnetics.com/prod } \\
\text { detail.asp?prod=RX038DCB-N52 }\end{array}$ & $\$ 13.91$ & 1 & $\$ 83.46$ \\
\hline $\begin{array}{l}\text { M4 } \times 25 \mathrm{~mm} \text { countersunk } \\
\text { machine screw }\end{array}$ & 6 & ea & $\begin{array}{l}\text { Secure } \\
\text { magnets } \\
\text { to } \\
\text { structure }\end{array}$ & $\begin{array}{l}\text { http://uww. boltdepot.com/Product- } \\
\text { Details.aspx?product }=5116\end{array}$ & $\$ 0.13$ & 1 & $\$ 0.78$ \\
\hline M4 flat washer & 6 & ea & $\begin{array}{l}\text { Secure } \\
\text { magnets } \\
\text { to } \\
\text { structure }\end{array}$ & $\begin{array}{l}\text { http://unw. boltdepot.com/Product- } \\
\text { Details.aspx?product }=4514\end{array}$ & $\$ 0.05$ & 1 & $\$ 0.30$ \\
\hline M4 hex nut & 6 & ea & $\begin{array}{l}\text { Secure } \\
\text { magnets } \\
\text { to } \\
\text { structure }\end{array}$ & $\begin{array}{l}\text { http://wnw. boltdepot.com/Product- } \\
\text { Details.aspx?product }=4774\end{array}$ & $\$ 0.05$ & 1 & $\$ 0.30$ \\
\hline $\begin{array}{l}\text { \#6 } \times 1 / 2 \text { " countersunk wood } \\
\text { screw }\end{array}$ & 3 & ea & $\begin{array}{l}\text { Mounting } \\
\text { cap to } \\
\text { cage }\end{array}$ & $\begin{array}{l}\text { http://wnw. boltdepot.com/Product- } \\
\text { Details. aspx?product=3953 }\end{array}$ & $\$ 0.08$ & 1 & $\$ 0.24$ \\
\hline & & & & & & $\begin{array}{l}\text { Total } \\
\text { Price }\end{array}$ & $\$ 85.86$ \\
\hline
\end{tabular}

The bills of materials for the tools demonstrated, a syringe pump, an extruder designed to accept products delivered in caulk tubes, and a micro mill spindle holder are provided in Tables 3, 4 and 5, respectively. 
Gerald Anzalone, Bas Wijnen, Joshua M. Pearce, (2015) "Multi-material additive and subtractive prosumer digital fabrication with a free and open-source convertible delta RepRap 3-D printer", Rapid Prototyping Journal, 21(5), pp.506 - 519. doi:http://dx.doi.org/10.1108/RPJ-09-2014-0113

Table 3. Bill of materials for syringe pump tool

\begin{tabular}{|c|c|c|c|c|c|c|c|}
\hline Description & Quantity & UOM & Function & Source & Price & $\begin{array}{l}\text { Sold in } \\
\text { units }\end{array}$ & Total Price \\
\hline Printed syringe pump body & 1 & ea & Motor end and bearing cage & Printed & & & $\$ 3.25$ \\
\hline Printed syringe retainer & 2 & ea & Secure syringe to pump & Printed & & & $\$ 0.28$ \\
\hline Printed syringe pump idler & 1 & ea & Idler end & Printed & & & $\$ 0.83$ \\
\hline Printed carriage & 1 & ea & Plunger carriage & Printed & & & $\$ 0.32$ \\
\hline Printed plunger trap & 1 & ea & Retainer plunger on carriage & Printed & & & $\$ 0.14$ \\
\hline Printed plunger retainer & 1 & ea & Retainer plunger on carriage & Printed & & & $\$ 0.05$ \\
\hline $625 z$ ball bearing & 2 & ea & Idler bearing & $\begin{array}{l}\text { http://www.amazon.com/Miniature- } \\
\text { Shielded-Deep-Groove- } \\
\text { Bearings/dp/B008LTIFX6/ref=sr_1_- } \\
\text { 2? } \\
\text { ie=UTF8\&qid=1405991639\&sr=8- } \\
\text { 2\&keywords=625z }\end{array}$ & $\$ 6.76$ & 5 & $\$ 2.70$ \\
\hline NEMA17 motor & 1 & ea & Drive & $\begin{array}{l}\text { http://wuw.kysanelectronics.com/P } \\
\text { roducts/Detail.php?recordID=7850 }\end{array}$ & $\$ 12.50$ & 1 & $\$ 12.50$ \\
\hline $6 \mathrm{~mm} \times 6 \mathrm{~mm}$ shaft coupling & 1 & ea & Drive-screw coupling & $\begin{array}{l}\text { http://mww.amazon.com/Motor- } \\
\text { Shaft-Coupler-Flexible- } \\
\text { Coupling/dp/BOODCAISM2/ref=sr_1 } \\
\text { 1? } \\
\text { ie=UTF8\&qid=1405991920\&sr=8- } \\
\text { 1\&keywords=5mm+shaft+coupling }\end{array}$ & $\$ 7.57$ & 1 & $\$ 7.57$ \\
\hline $\begin{array}{l}\text { M3 x 10mm socket head } \\
\text { cap screw }\end{array}$ & 6 & ea & $\begin{array}{l}\text { Guide rod clamps and } \\
\text { plunger retainer }\end{array}$ & $\begin{array}{l}\text { http://wnw.boltdepot.com/Product- } \\
\text { Details.aspx?product=6380 }\end{array}$ & $\$ 0.06$ & 1 & $\$ 0.36$ \\
\hline $\begin{array}{l}\text { M3 } \times 14 \mathrm{~mm} \text { socket head } \\
\text { cap screw }\end{array}$ & 4 & ea & Syringe retainer screws & $\begin{array}{l}\text { http://www.mcmaster.com/\#91292a } \\
\text { 027/=sxuvqf }\end{array}$ & $\$ 5.60$ & 100 & $\$ 0.22$ \\
\hline $\begin{array}{l}\text { M3 } \times 16 \mathrm{~mm} \text { socket head } \\
\text { cap screw }\end{array}$ & 4 & ea & Motor mount screws & $\begin{array}{l}\text { http://www.boltdepot.com/Product- } \\
\text { Details.aspx?product=6382 }\end{array}$ & $\$ 0.06$ & 1 & $\$ 0.24$ \\
\hline $\begin{array}{l}\text { M3 } \times 20 \mathrm{~mm} \text { socket head } \\
\text { cap screw }\end{array}$ & 3 & ea & Attaching ball cage & $\begin{array}{l}\text { http://wnww.boltdepot.com/Product- } \\
\text { Details.aspx?product=6383 }\end{array}$ & $\$ 0.07$ & 1 & $\$ 0.21$ \\
\hline M3 hex nut & 13 & ea & Various & $\begin{array}{l}\text { http://www.boltdepot.com/Product- } \\
\text { Details.aspx?product=4773 }\end{array}$ & $\$ 0.05$ & 1 & $\$ 0.65$ \\
\hline M5 threaded rod & 0.2 & $\mathrm{~m}$ & Drive & $\begin{array}{l}\text { http://www.mcmaster.com/\#90024a } \\
\text { 060/=sxuwfc }\end{array}$ & $\$ 9.24$ & 1 & $\$ 1.85$ \\
\hline M5 hex nut & 3 & ea & Captive nut, idler jam nuts & $\begin{array}{l}\text { http://wnw.boltdepot.com/Product- } \\
\text { Details.aspx?product=4775 }\end{array}$ & $\$ 0.05$ & 1 & $\$ 0.15$ \\
\hline $6 \mathrm{~mm}$ A2 tool steel & 0.4 & $m$ & Guide rod & $\begin{array}{l}\text { http://wnw.mcmaster.com/\#8116k3 } \\
\text { 5/=sxuwsm }\end{array}$ & $\$ 9.54$ & 0.9144 & $\$ 4.17$ \\
\hline LM6UU linear bearing & 2 & ea & Carriage & $\begin{array}{l}\text { http://www.amazon.com/10pcs- } \\
\text { Linear-Bearing-Bushing- } \\
\text { linear/dp/B00AGCIS74/ref=sr_1_2? } \\
\text { ie=UTF8\&qid=1405992161\&sr=8- } \\
\text { 2\&keywords=Im6uu }\end{array}$ & $\$ 15.50$ & 10 & $\$ 3.10$ \\
\hline $\begin{array}{l}\text { 1" diameter } \mathrm{G} 25 \text { ball } \\
\text { bearings }\end{array}$ & 3 & ea & Magnetic mount & $\begin{array}{l}\text { http://www.amazon.com/gp/product } \\
\text { /B000FMULLG/ref=oh_details_o04 } \\
\text { s00_i00?ie=UTF8\&psc=1 }\end{array}$ & $\$ 13.75$ & 10 & $\$ 4.13$ \\
\hline & & & & & & \begin{tabular}{l|} 
Total \\
Price
\end{tabular} & $\$ 42.71$ \\
\hline
\end{tabular}


Gerald Anzalone, Bas Wijnen, Joshua M. Pearce, (2015) "Multi-material additive and subtractive prosumer digital fabrication with a free and open-source convertible delta RepRap 3-D printer", Rapid Prototyping Journal, 21(5), pp.506 - 519. doi:http://dx.doi.org/10.1108/RPJ-09-2014-0113

Table 4. Bill of materials for caulk extruder

\begin{tabular}{|c|c|c|c|c|c|c|c|}
\hline Description & Quantity & UOM & Function & Source & Price & $\begin{array}{l}\text { Sold in } \\
\text { units }\end{array}$ & \begin{tabular}{|l|} 
Total \\
Price
\end{tabular} \\
\hline Printed 5.45:1 planetary gear & 3 & ea & & Printed & & & $\$ 0.14$ \\
\hline Printed 5.45:1 sun gear & 1 & ea & & Printed & & & $\$ 0.23$ \\
\hline $\begin{array}{l}\text { Printed 5.45:1 input annulus } \\
\text { gear }\end{array}$ & 1 & ea & & Printed & & & $\$ 0.46$ \\
\hline $\begin{array}{l}\text { Printed 5.45:1 output annulus } \\
\text { gear }\end{array}$ & 1 & ea & & Printed & & & $\$ 0.55$ \\
\hline $\begin{array}{l}\text { Printed 5.45:1 planetary } \\
\text { carrier }\end{array}$ & & ea & & Printed & & & $\$ 0.46$ \\
\hline Printed gearbox motor mount & 1 & ea & & Printed & & & $\$ 0.69$ \\
\hline Printed gearbox output end & 1 & ea & & Printed & & & $\$ 0.92$ \\
\hline Printed piston & 1 & ea & & Printed & & & $\$ 0.37$ \\
\hline Printed 23-tooth gear & 1 & ea & & Printed & & & $\$ 0.92$ \\
\hline Printed 49-tooth gear & 1 & ea & & Printed & & & $\$ 1.29$ \\
\hline Printed lead screw retainer & 1 & ea & & Printed & & & $\$ 0.69$ \\
\hline Printed caulk idler end & 1 & ea & & Printed & & & $\$ 2.53$ \\
\hline Printed caulk output end & 1 & ea & & Printed & & & $\$ 2.07$ \\
\hline Printed caulk tube backer & 1 & ea & & Printed & & & $\$ 1.01$ \\
\hline Copper tube adapter & 1 & ea & & Hardware store & & & $\$ 4.00$ \\
\hline NEMA17 motor & 1 & ea & & $\begin{array}{l}\text { http://www.kysanelectronics.com/Products/ } \\
\text { Detail.php?recordID=7850 }\end{array}$ & $\$ 12.50$ & 1 & $\$ 12.50$ \\
\hline 1/4" hose clamp & 2 & ea & & Hardware store & $\$ 0.79$ & 1 & $\$ 1.58$ \\
\hline M8 threaded rod & 1.5 & $\mathrm{~m}$ & & $\begin{array}{l}\text { http://www.mcmaster.com/\#90024a080/=sx } \\
\text { uxk0 }\end{array}$ & $\$ 10.50$ & 1 & $\$ 15.75$ \\
\hline M8 hex nut & 10 & ea & & $\begin{array}{l}\text { http://www.boltdepot.com/Product- } \\
\text { Details.aspx?product=4778 }\end{array}$ & $\$ 0.13$ & 1 & $\$ 1.30$ \\
\hline M8 flat washer & 4 & ea & & $\begin{array}{l}\text { http://www.boltdepot.com/Product- } \\
\text { Details.aspx?product }=4518\end{array}$ & $\$ 0.08$ & 1 & $\$ 0.32$ \\
\hline $608 z z$ ball bearing & 3 & ea & & $\begin{array}{l}\text { http://www. amazon.com/Set-608ZZ-Radial- } \\
\text { Bearings- } \\
\text { Printer/dp/B00C7BJTU2/ref=sr_1_3? } \\
\text { s=industrial\&ie=UTF8\&qid=1405992309\&sr } \\
\text { =1-3\&keywords=608zz }\end{array}$ & $\$ 6.00$ & 9 & $\$ 2.00$ \\
\hline 2" PVC pipe, split lengthwise & 0.21 & $\mathrm{~m}$ & & $\begin{array}{l}\text { http://www.homedepot.com/p/Unbranded-2- } \\
\text { in-X-10-ft-PVC-Sch-40-Plain-End-Pipe- } \\
\text { 531137/100161954 }\end{array}$ & $\$ 5.06$ & 3.04801 & $\$ 0.35$ \\
\hline 2" hose clamp & 5 & ea & & Hardware store & $\$ 1.05$ & 1 & $\$ 5.25$ \\
\hline $624 z$ ball bearing & 3 & ea & & $\begin{array}{l}\text { http://www.amazon.com/10-Bearing-624ZZ- } \\
\text { Shielded- } \\
\text { 4x13x5/dp/B002BBH45A/ref=sr_1_1? } \\
\text { s=industrial\&ie=UTF8\&qid=1405992358\&sr } \\
=1-1 \& k e y w o r d s=624 z z\end{array}$ & $\$ 15.38$ & 10 & $\$ 4.61$ \\
\hline $\begin{array}{l}\text { M4 x 16mm socket head cap } \\
\text { screw }\end{array}$ & 3 & ea & & $\begin{array}{l}\text { http://www.boltdepot.com/Product- } \\
\text { Details.aspx?product=6396 }\end{array}$ & $\$ 0.09$ & 1 & $\$ 0.27$ \\
\hline M4 flat washer & 9 & ea & & $\begin{array}{l}\text { http://www.boltdepot.com/Product- } \\
\text { Details.aspx?product=4514 }\end{array}$ & $\$ 0.05$ & 1 & $\$ 0.45$ \\
\hline M4 nylock nut & 3 & ea & & $\begin{array}{l}\text { http://www.boltdepot.com/Product- } \\
\text { Details.aspx?product=4793 }\end{array}$ & $\$ 0.06$ & 1 & $\$ 0.18$ \\
\hline M8 x 60mm hex head bolt & 1 & ea & & $\begin{array}{l}\text { http://www.boltdepot.com/Product- } \\
\text { Details.aspx?product=5821 }\end{array}$ & $\$ 0.73$ & 1 & $\$ 0.73$ \\
\hline $\begin{array}{l}\text { M3 } \times 30 \mathrm{~mm} \text { socket head cap } \\
\text { screw }\end{array}$ & 4 & ea & Gearbox & $\begin{array}{l}\text { http://www.boltdepot.com/Product- } \\
\text { Details.aspx?product=6385 }\end{array}$ & $\$ 0.10$ & 1 & $\$ 0.40$ \\
\hline M3 flat washer & 8 & ea & & $\begin{array}{l}\text { http://www.boltdepot.com/Product- } \\
\text { Details.aspx?product=4513 }\end{array}$ & $\$ 0.05$ & 1 & $\$ 0.40$ \\
\hline M3 hex nut & 7 & ea & & $\begin{array}{l}\text { http://www.boltdepot.com/Product- } \\
\text { Details.aspx?product=4773 }\end{array}$ & $\$ 0.05$ & 1 & $\$ 0.35$ \\
\hline $\begin{array}{l}\text { M3 } \times 10 \mathrm{~mm} \text { socket head cap } \\
\text { screw }\end{array}$ & 4 & ea & Motor mount & $\begin{array}{l}\text { http://www.boltdepot.com/Product- } \\
\text { Details.aspx?product=6380 }\end{array}$ & $\$ 0.06$ & 1 & $\$ 0.24$ \\
\hline M8 hex nut & 15 & ea & & $\begin{array}{l}\text { http://www.boltdepot.com/Product- } \\
\text { Details.aspx?product=4778 }\end{array}$ & $\$ 0.13$ & 1 & $\$ 1.95$ \\
\hline M8 flat washer & 11 & ea & & $\begin{array}{l}\text { http://www.boltdepot.com/Product- } \\
\text { Details.aspx?product }=4518\end{array}$ & $\$ 0.08$ & 1 & $\$ 0.88$ \\
\hline $\begin{array}{l}\text { M3 } \times 20 \mathrm{~mm} \text { socket head cap } \\
\text { screw }\end{array}$ & 3 & ea & $\begin{array}{l}\text { Attaching ball } \\
\text { cage }\end{array}$ & $\begin{array}{l}\text { http://www.boltdepot.com/Product- } \\
\text { Details.aspx?product=6383 }\end{array}$ & $\$ 0.07$ & 1 & $\$ 0.21$ \\
\hline 1" diameter G25 ball bearings & 3 & ea & Magnetic mount & $\begin{array}{l}\text { http://www.amazon.com/gp/product/B000F } \\
\text { MULLG/ref=oh_details_004_s00_i00? } \\
\text { ie=UTF8\&psc=1 }\end{array}$ & $\$ 13.76$ & 10 & $\$ 4.13$ \\
\hline & & & & & & \begin{tabular}{|l|} 
Total \\
Price
\end{tabular} & $\$ 70.18$ \\
\hline
\end{tabular}


Gerald Anzalone, Bas Wijnen, Joshua M. Pearce, (2015) "Multi-material additive and subtractive prosumer digital fabrication with a free and open-source convertible delta RepRap 3-D printer", Rapid Prototyping Journal, 21(5), pp.506 - 519. doi:http://dx.doi.org/10.1108/RPJ-09-2014-0113

Table 5. Bill of materials for micro mill spindle mount

\begin{tabular}{|c|c|c|c|c|c|c|c|}
\hline Description & Quantity & UOM & Function & Source & Price & $\begin{array}{l}\text { Sold } \\
\text { in } \\
\text { units }\end{array}$ & $\begin{array}{l}\text { Total } \\
\text { Price }\end{array}$ \\
\hline Printed spindle clamp & 1 & ea & & Printed & & & $\$ 1.61$ \\
\hline Printed vacuum attachment & 1 & ea & & Printed & & & $\$ 0.69$ \\
\hline Printed bearing cage & 1 & ea & & Printed & & & $\$ 1.84$ \\
\hline Mill spindle & 1 & ea & & $\begin{array}{l}\text { http://www.angelfire.com/az2/prof } \\
\text { f/ }\end{array}$ & $\$ 92.00$ & 1 & $\$ 92.00$ \\
\hline $\begin{array}{l}\text { M4 x 16mm socket head cap } \\
\text { screw }\end{array}$ & 1 & ea & Spindle clamp & $\begin{array}{l}\text { http://www.boltdepot.com/Product } \\
\text {-Details.aspx?product=6396 }\end{array}$ & $\$ 0.09$ & 1 & $\$ 0.09$ \\
\hline M4 hex nut & 1 & ea & Spindle clamp & $\begin{array}{l}\text { http://www.boltdepot.com/Product } \\
\text {-Details.aspx?product }=4774\end{array}$ & $\$ 0.05$ & 1 & $\$ 0.05$ \\
\hline M4 flat washer & 1 & ea & Spindle clamp & $\begin{array}{l}\text { http://www.boltdepot.com/Product } \\
\text {-Details.aspx?product=4514 }\end{array}$ & $\$ 0.05$ & 1 & $\$ 0.05$ \\
\hline $\begin{array}{l}\text { M3 x 20mm socket head cap } \\
\text { screw }\end{array}$ & 3 & ea & Attaching bearing cage & $\begin{array}{l}\text { http://www.boltdepot.com/Product } \\
\text {-Details.aspx?product=6383 }\end{array}$ & $\$ 0.07$ & 1 & $\$ 0.21$ \\
\hline M3 hex nut & 3 & ea & Attaching bearing cage & $\begin{array}{l}\text { http://www.boltdepot.com/Product } \\
\text {-Details.aspx?product=4773 }\end{array}$ & $\$ 0.05$ & 1 & $\$ 0.15$ \\
\hline M3 flat washer & 3 & ea & Attaching bearing cage & $\begin{array}{l}\text { http://www.boltdepot.com/Pro } \\
\text { duct-Details.aspx? } \\
\text { product=4513 }\end{array}$ & $\$ 0.05$ & 1 & $\$ 0.15$ \\
\hline $\begin{array}{l}\text { M3 x 10mm socket head cap } \\
\text { screw }\end{array}$ & 1 & ea & Vacuum attachment clamp & $\begin{array}{l}\text { http://www.boltdepot.com/Product } \\
\text {-Details.aspx?product=6380 }\end{array}$ & $\$ 0.06$ & 1 & $\$ 0.06$ \\
\hline 1" diameter $\mathrm{G} 25$ ball bearings & 3 & ea & Magnetic mount & $\begin{array}{l}\text { http://www.amazon.com/gp/produ } \\
\text { ct/B000FMULLG/ref=oh_details_ } \\
\text { o04_s00_i00?ie=UTF8\&psc=1 }\end{array}$ & $\$ 13.76$ & 10 & $\$ 4.13$ \\
\hline & & & & & & $\begin{array}{l}\text { Total } \\
\text { Price }\end{array}$ & $\$ 101.03$ \\
\hline
\end{tabular}

Finally, a 3-D printable end effector was designed that permits easy swapping of tools using a smaller version of the fixedtool mount. Tool holders for fixing pens and a vinyl cutter were designed and printed. Bills of material for the end effector and tool holders is provided in Table 6 and Table 7, respectively.

The entire software tool chain is open-source and freely available. Franklin printer firmware (Wijnen, et al., 2015) is configured for each of the tools, setting tool offset, extrusion rate and other parameters necessary. Three dimensional models for printing were sliced with Cura (software.ultimaker.com). Printed circuit boards were designed with kicad (kicad-pcb.org) and converted to g-code using pcb2gcode (sourceforge.net/projects/pcb2gcode/). Scalable vector graphics (SVG) line art files were converted to g-code using Inkscape (inkscape.org) and the Gcodetools plugin (github.com/cnc-club/gcodetools) for pen plotting, vinyl cutting and eventually for engraving. 
Gerald Anzalone, Bas Wijnen, Joshua M. Pearce, (2015) "Multi-material additive and subtractive prosumer digital fabrication with a free and open-source convertible delta RepRap 3-D printer", Rapid Prototyping Journal, 21(5), pp.506 - 519. doi:http://dx.doi.org/10.1108/RPJ-09-2014-0113

Table 6. Bill of materials for tool effector

\begin{tabular}{|c|c|c|c|c|c|c|c|}
\hline Description & Quantity & UOM & Function & Source & Price & $\begin{array}{l}\text { Sold in } \\
\text { units }\end{array}$ & $\begin{array}{l}\text { Total } \\
\text { Price }\end{array}$ \\
\hline $\begin{array}{l}\text { Printed tool } \\
\text { end effector }\end{array}$ & & ea & $\begin{array}{l}\text { Effector that } \\
\text { accepts tools }\end{array}$ & Printed & & & 2.07 \\
\hline $\begin{array}{l}3 / 8 \text { " ring } \\
\text { magnet }\end{array}$ & 12 & & $\begin{array}{l}\text { Magnetic tool } \\
\text { mounts and } \\
\text { ball joints }\end{array}$ & $\begin{array}{l}\text { https://www.kjmagnetics.co } \\
\text { m/proddetail.asp? } \\
\text { prod=R622CS-P }\end{array}$ & $\$ 1.17$ & 2 & $\$ 7.02$ \\
\hline & & & & & \multicolumn{2}{|c|}{ Total Price } & $\$ 9.09$ \\
\hline
\end{tabular}


Table7. Bill of materials for tool effector spring mount tool

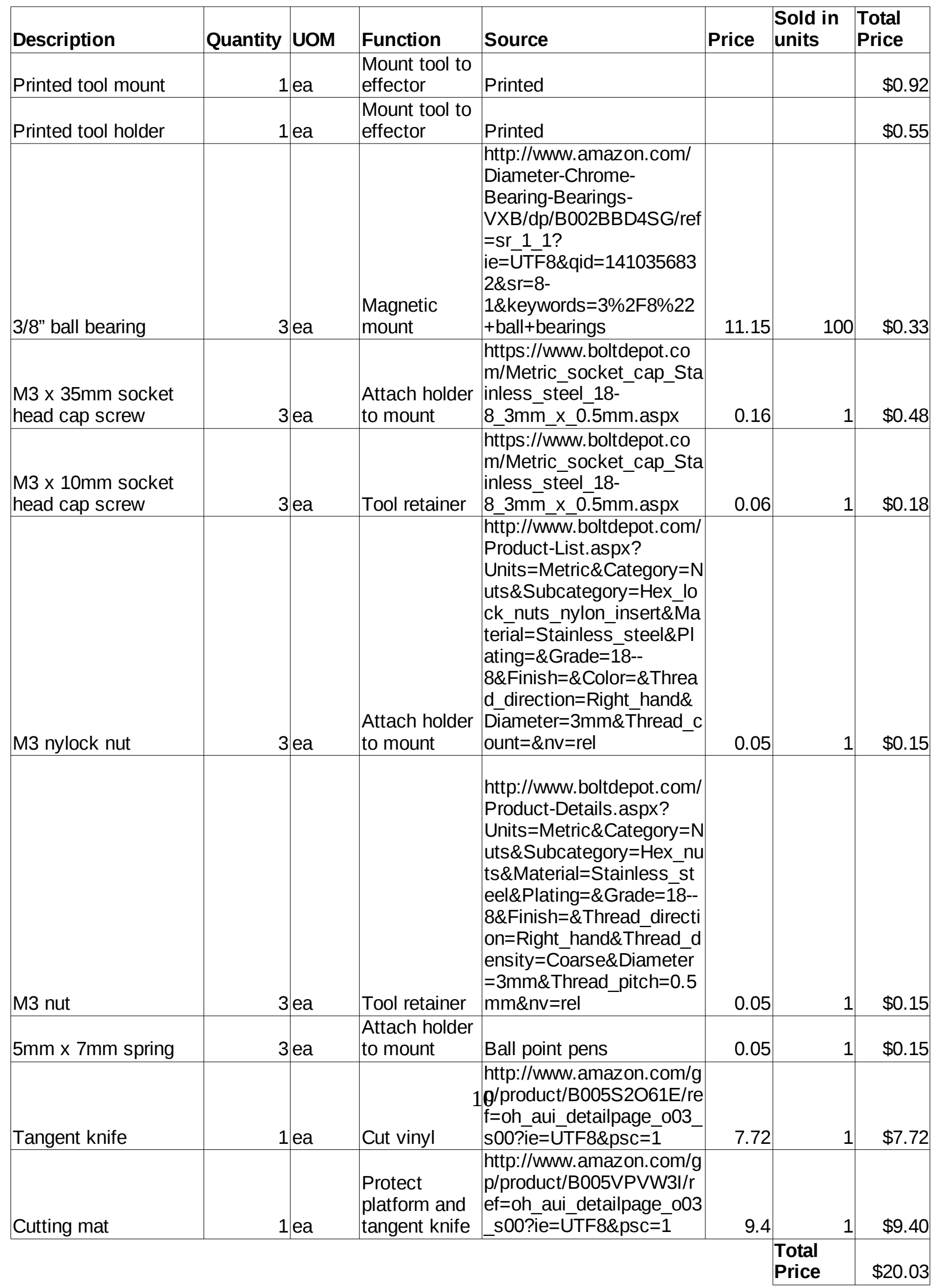


Gerald Anzalone, Bas Wijnen, Joshua M. Pearce, (2015) "Multi-material additive and subtractive prosumer digital fabrication with a free and open-source convertible delta RepRap 3-D printer", Rapid Prototyping Journal, 21(5), pp.506 - 519. doi:http://dx.doi.org/10.1108/RPJ-09-2014-0113

\section{Results}

The MOST Delta RepRap was successfully converted to a convertible RepRap using the materials shown in Table 1 and 2 while still maintaining the ability to print with plastic in the stationary substrate configuration as shown in Figure 2 . In this configuration the RepRap functions as a standard polymer 3-D printer, the extremely small increase in the mass of the double sided replacement carriages had no impact on speed, resolution or reproducibility of PLA prints.

Figure 2. Digital Photograph of Assembled Convertible RepRap in mobile tool mode with 3-D printer end effector for printing thermo polymers with conventional fused filament fabrication.

In this configuration, the printer can be used to produce a number of other tools including the plastic parts listed in Tables 3-7. Tools too large to fit on the moving end effector were designed with steel ball bearings that mate with a magnetic base attached to the aluminum vertical board. The magnetic mount makes for rapid and easy tool interchange. This tool interchange is a manual operation.
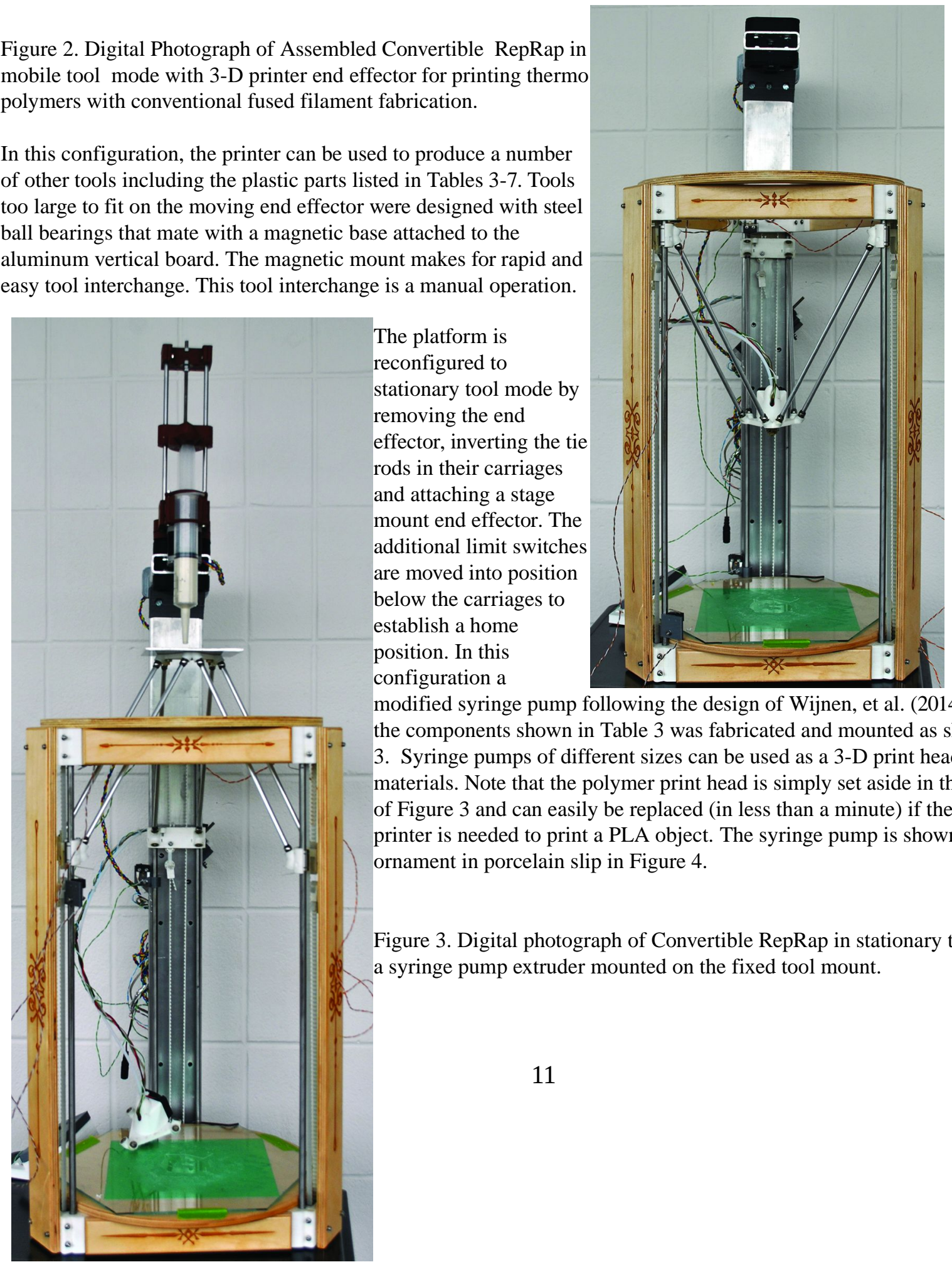

modified syringe pump following the design of Wijnen, et al. (2014) consisting of the components shown in Table 3 was fabricated and mounted as shown in Figure 3. Syringe pumps of different sizes can be used as a 3-D print head for viscous materials. Note that the polymer print head is simply set aside in the bottom left of Figure 3 and can easily be replaced (in less than a minute) if the convertible printer is needed to print a PLA object. The syringe pump is shown extruding an ornament in porcelain slip in Figure 4.

Figure 3. Digital photograph of Convertible RepRap in stationary tool mode with a syringe pump extruder mounted on the fixed tool mount. 
Figure 4. Close up digital photograph of the Convertible RepRap with the syringe pump extruder operating to produce an ornament made from porcelain slip.

For larger viscous prints, using common materials an extruder designed to accept standard-size caulk tubes ("caulkstruder", BOM in Table 4) containing a variety of viscous sealants and cements was fabricated. The caulkstruder mounted on the

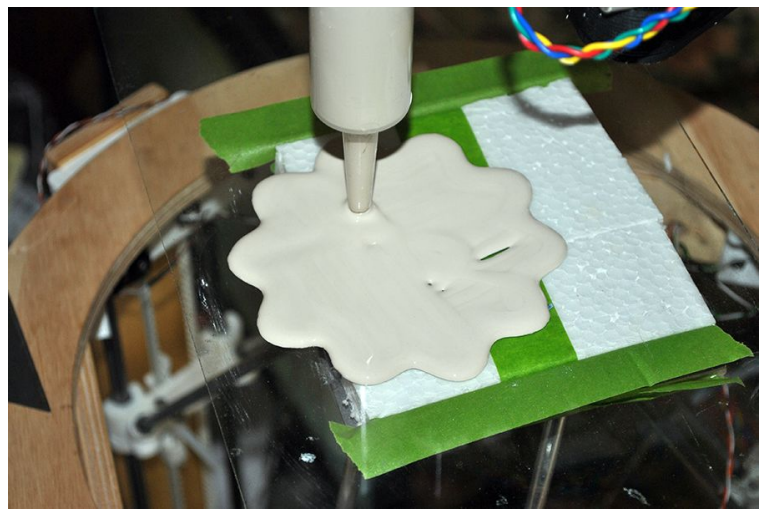
convertible printer is shown in Figure 5. This is a significantly larger and heavier tool meant to print in any material available in hardware stores in standard caulk tubes. In the inset of Figure 5, the caulk extruder, with white silicone caulk loaded, is shown printing a custom silicone orthotic (Nettleton, 2013).

Figure 5. Digital photograph of the Convertible RepRap with the caulkextruder mounted. The inset shows a custom orthotic being printed in silicone caulk. The caulk extruder tool is attached to the fixed tool mount.

All of the tools were designed to use as many 3-D printed parts as possible, in keeping with the RepRap philosophy. All of the printed parts were produced on the machine they were to be attached to and only require hardware ('vitamins' in RepRap parlance). For example, note the 3-D printed (printed in the stationary substrate configuration) planetary gearbox in the upper-right hand corner of Figure 5 is also an open-source parametric file that can be customized using OpenSCAD (MTU-MOST, 2014).

The convertible Delta RepRap can also be used for subtractive manufacturing and prototyping. In Figure 6, the results of assembling the components listed in Table 5 are shown to make a lightweight micro mill. The micro mill spindle is shown placed the tool mount being used to produce the circuit boards shown in inset of Figure 6.

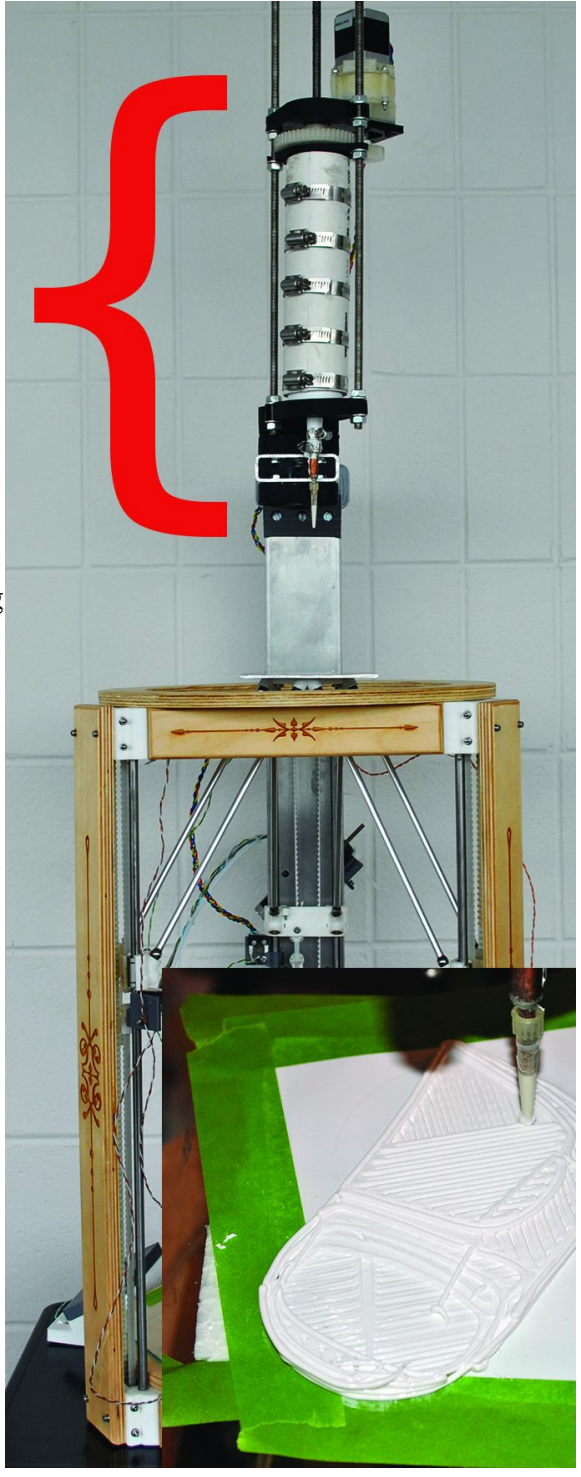


Gerald Anzalone, Bas Wijnen, Joshua M. Pearce, (2015) "Multi-material additive and subtractive prosumer digital fabrication with a free and open-source convertible delta RepRap 3-D printer", Rapid Prototyping Journal, 21(5), pp.506 - 519. doi:http://dx.doi.org/10.1108/RPJ-09-2014-0113

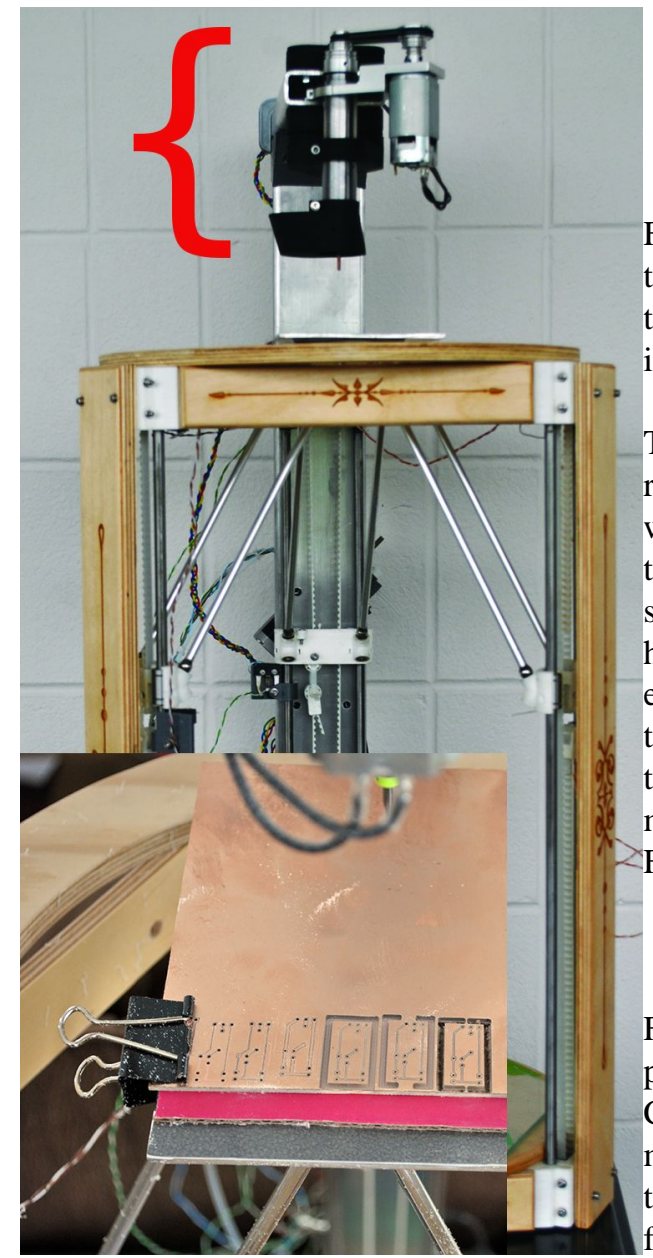

Figure 6. Digital photograph of the Convertible RepRap with the micro mill tool mounted. Inset: Digital photograph of printed circuit boards milled with the micro mill tool. The micro mill spindle is just visible in the top of the inset.

The platform can also be used as other conventionally 2-D tools when it is reconfigured to mobile tool mode with the tool end effector in place on the working end of the tie rods. A tool end effector for mounting small, low mass tools on the moving end effector was designed using a similar, but much smaller magnetic mount (Table 6). A spring-loaded tool mount (Table 7) for holding cylindrical tools was fabricated and tested for functionality. For example, the spring mounted tool was outfitted with a tangent knife and a tacky cutting mat was placed on the glass build platform. Vinyl was secured to the mat and patterns were cut as shown in Figure 7. Using the same setup, a marking pen can be affixed to be used as plotter for graphics as shown in Figure 8.

Figure 7. Digital photograph of the Convertible RepRap in mobile tool mode with the tool effector in place fitted with a tangent knife cutting a pattern in pressure sensitive adhesive-backed vinyl.

Figure 8. Digital photograph of the tool effector fitted with a permanent marker plotting a graphic on plain, white paper.

In all cases, the platform performed well as compared to its single-purpose, commercial counterparts, producing useful and usable products. Of particular merit was the fact that the platform performed the different functions predictably, i.e. when converting from fixed tool mode to mobile tool mode for 3-D printing, which requires inversion of all tie rods, no adjustments were required and the quality of the printed part was unaffected. The use of magnetic mounts

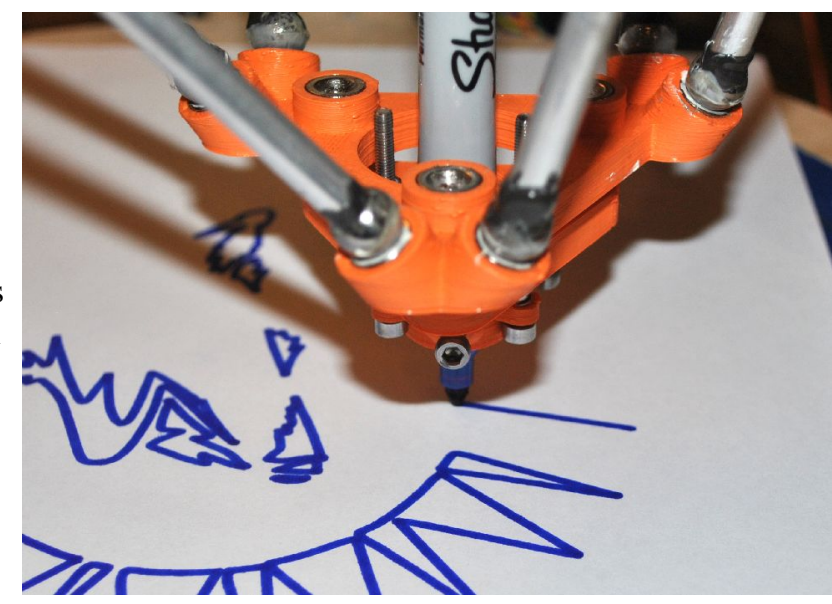
greatly facilitated interchanging tools and conversion from stationary tool to mobile tool modes.

Table 8 summarizes the functions demonstrated by the convertible delta RepRap and compares the cost of the function 
provided by the open-source RepRap to commercial equivalent devices. It should be noted that the plotter is not listed as this technology has been largely been replaced by conventional 2-D printers. The total cost of the convertible delta RepRap with all of the tools shown in Figures 2-8 is less than $\$ 1,000$, which is striking compared to even a single purpose proprietary tool. The total capital expenditure reductions when using the convertible delta RepRap compared to single-purpose tools for only the functionality demonstrated in this study are between $\$ 7,000$ and $\$ 29,000$. This represents a savings to prosumers of between 90 and $97 \%$. It should be pointed out that smaller RepRap 3-D printers can be purchased for under $\$ 500$, that the delta RepRap shown here is not only comparable in cost for thermo polymer printing alone, but also capable of the full set of additional functionalities (e.g PCB milling) demonstrated in Figures 2-8. 
Gerald Anzalone, Bas Wijnen, Joshua M. Pearce, (2015) "Multi-material additive and subtractive prosumer digital fabrication with a free and open-source convertible delta RepRap 3-D printer", Rapid Prototyping Journal, 21(5), pp.506 - 519. doi:http://dx.doi.org/10.1108/RPJ-09-2014-0113

Table 8. Comparison of the functions demonstrated by the Convertible Delta RepRap to commercial equivalents and the associated costs.

\begin{tabular}{|c|c|c|c|c|}
\hline Function & Proprietary Equivalent & $\begin{array}{l}\text { CAPEX Cost } \\
\text { (US\$) }\end{array}$ & $\begin{array}{l}\text { Material Cost } \\
\text { (US\$) }\end{array}$ & Notes \\
\hline $\begin{array}{l}\text { Thermoplastic 3-D } \\
\text { Printing }\end{array}$ & $\begin{array}{l}\text { Makerbot Replicator } \\
\text { Stratasys } \mu \text { Print SE Plus }\end{array}$ & $\begin{array}{l}\$ 2,900 \\
\sim \$ 20,000\end{array}$ & $\begin{array}{l}\$ 53 / \mathrm{kg} \\
\sim \$ 200 / \mathrm{kg}\end{array}$ & $\begin{array}{l}\text { http://www.makerb } \\
\text { ot.com/ } \\
\text { http://www.stratasy } \\
\text { s.com/ }\end{array}$ \\
\hline Paste 3-D printer & Foodini & $\$ 1,000$ & N/A & $\begin{array}{l}\text { http://www.natural } \\
\text { machines.com/ }\end{array}$ \\
\hline $\begin{array}{l}\text { Vinyl Cutter/ } \\
\text { Plotter }\end{array}$ & Seiki SK375T & $\$ 230$ & $\$ 10 / \mathrm{m}^{2}$ & $\begin{array}{l}\text { http://www.joyfay.c } \\
\text { om/brand-new-375- } \\
\text { mm-15-lcd-sign- } \\
\text { sticker-vinyl-cutter- } \\
\text { cutting-plotter- } \\
\text { artcut-sk375t.html? } \\
\text { gclid=CJeB7JyZ18 } \\
\text { ACFRQdaQod51k } \\
\text { AjA }\end{array}$ \\
\hline Milling & $\begin{array}{l}\text { LPKF ProtoMat } \\
\text { MITS Eleven Lab } \\
\text { Colinbus PCBBox }\end{array}$ & $\begin{array}{l}\$ 8750 \\
\$ 8495 \\
\$ 4,500 \\
(3500 €)\end{array}$ & N/A & \\
\hline Total & None & $\begin{array}{l}\$ 8,600- \\
\$ 30,00\end{array}$ & & \\
\hline $\begin{array}{l}\text { Convertible Delta } \\
\text { RepRap w/ all } \\
\text { tools }\end{array}$ & & $\$ 825$ & & \\
\hline $\begin{array}{l}\text { Savings from } \\
\text { RepRap } \\
\text { Agglomeration }\end{array}$ & & $\begin{array}{l}\$ 7,775- \\
\$ 29,175\end{array}$ & & \\
\hline
\end{tabular}


Gerald Anzalone, Bas Wijnen, Joshua M. Pearce, (2015) "Multi-material additive and subtractive prosumer digital fabrication with a free and open-source convertible delta RepRap 3-D printer", Rapid Prototyping Journal, 21(5), pp.506 - 519. doi:http://dx.doi.org/10.1108/RPJ-09-2014-0113

\section{Discussion}

As with most comparisons of multi-purpose tools to the purpose-built tools they are designed to compete with, shortcomings are apparent. Although, the convertible RepRap performed adequately at all tasks considered in this evaluation, it is clear refinements can be made and can be expected following the open source model of technical development. In some cases the RepRap, when tuned, can even out perform the commercial equivalents. This was demonstrated previously in a study of the tensile strength of 3-D printed parts using PLA and ABS (Tymrak, et al., 2014).

Of the applications investigated here, PCB milling proved to be the most challenging. This can be seen in the micrographs of printed circuit boards produced by a commercial PCB mill and the convertible platform shown in Figure 9a and 9b, respectively, demonstrate more chattering is occurring with the convertible RepRap.
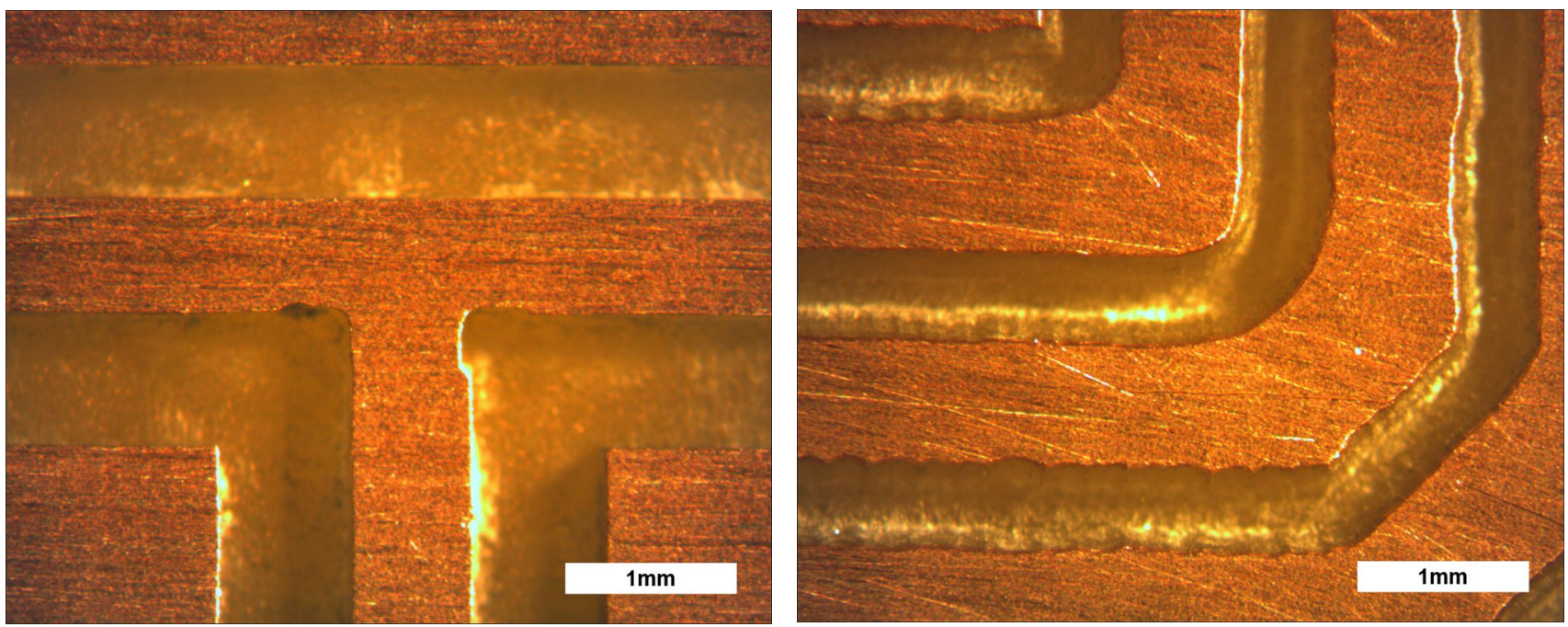

Figure 9. Digital micrographs of traces milled in printed circuit boards produced by a) a commercial PCB milling machine and b) the Convertible RepRap.

However, considering the very preliminary nature of this evaluation and the fact that the platform is an order of magnitude less expensive than most commercial, purpose-built PCB mills, even with all of the additional tools evaluated, it is clear that the convertible RepRap is an exceptional value. With this new functionality prosumers may improve technical sophistication and begin to fabricate smart objects or electrically functional objects from the use of entry level open-source making machines.

This study confirms the high-value of custom distributed manufacturing (or home manufacturing) (Wittbrodt, et al., 2013). For example, the pair of custom orthotics in the inset of Figure 5 can cost consumers over $\$ 500$ a pair. Printing out only two pairs with a tube of caulk can economically justify the entire convertible RepRap and all of the additional tools. The flexibility afforded by the platform is unparalleled and the opportunity for continuous improvement of the open-source design ensures that it will be refined and that additional capabilities will be added to it's already significant list of features, ensuring that the platform remains valuable. Unlike digital designs for objects that are not capable of self-replication the value of the RepRap designs is difficulty to quantify (Pearce, 2015). However, even modest conservative estimates of the number of applications of this process would result in substantial economic value (Pearce, 2015).

From the perspective of driving innovation, the affordability of the convertible RepRap and its intrinsic extensibility allows both professionals and tinkerer-makers to explore their ideas with much greater freedom. RepRaps currently dominate entrylevel 3-D printing in the maker community (Moilanen \& Vadén, 2012). As the many RepRap variants all share a common 
Gerald Anzalone, Bas Wijnen, Joshua M. Pearce, (2015) "Multi-material additive and subtractive prosumer digital fabrication with a free and open-source convertible delta RepRap 3-D printer", Rapid Prototyping Journal, 21(5), pp.506 - 519. doi:http://dx.doi.org/10.1108/RPJ-09-2014-0113

core technical infrastructure, the tools (e.g. print heads) can be shared between them. This is a major advantage over closed source development of rapid prototyping and manufacturing tools, which are normally designed specifically to avoid functioning with competitors products. Given the platform's predictable behavior, workflows for producing different items can be optimized, compressing time for prototyping and allowing more rapid evaluation of concepts. With this platform, more people can do more prototyping faster than might be possible with multiple, conventional, purpose-built tools. In addition, prosumers can make more expensive, more complicated products as they harness more sophisticated means of production with entry-level investments. The commercial behavior observed in conventional RepRap 3-D printing, where open-source tools first radically reduced costs and then commercial variants began to lower costs and provide solutions, can be expected to occur in multi-machines as well. Already commercial, multi-purpose prototypers are being developed (Molitch-Hou, 2013; 2014; ZeGo Robotics, 2014).

As Campbell, Bourell \& Gibson (2012) point out when discussing the future of additive manufacturing: "where materials advances are foreseen to occur will be by do-it-yourselfers", which will drive an increase in demand and the concomitant reduction in feedstock costs. They believe that this demand will accelerate the entry of major suppliers into the marketplace with their new and improved materials and even more functionality. This prediction can already be seen to be coming true in the fourth column of Table 8. Proprietary feedstock for fused filament fabrication 3-D printers costs about $\$ 200 / \mathrm{kg}$ and proprietary prosumer filament $\$ 53 / \mathrm{kg}$, while generic filament currently costs about $\$ 35 / \mathrm{kg}$ and recyclebot filament costs less than $\$ 0.10 / \mathrm{kg}$ for makers producing it themselves from their own household waste. Groups such as the Plastic Bank and the Ethical Filament Foundation have already begun to work with major materials suppliers to develop mass-production of social plastic/ethical filament gathered by waste pickers in the developing world (Feeley, et al. 2014). Multi-purpose tools

like the one described here will enable prototyping and manufacturing with an even wider range of materials, thus providing the opportunity to expand this form of high-value recycling to additional materials.

\section{Future Work}

The functionality of the standard delta RepRap has been greatly expanded by this study, yet there are still many applications that are left for future development. Using the same tool head holder as the plotter the system could be used for engraving a variety of materials. The system can also be used as a bioprinter (Hock et al., 2014) or food printer (Cohen et al., 2009) by overcoming former limitations of standard RepRaps. For example, with the syringe head it could be used to decorate cakes and cupcakes, make custom pancakes, sandwiches or yet unknown digital culinary masterpieces. The platform can be utilized by artists, for personalization or for prototyping logos on products. For example, it could be used to paint conventional pictures, make stop action movies, or the standard hot end could be used without filament as a wood burner. By developing the ability to map arbitrary substrates to tolerate complex geometries, the system could be used to prototype skins on 3-D objects made for decorative or functional purposes. Considerable work is still needed to understand the process parameter effects on standard RepRaps (Lanzotti, Martorelli, \& Staiano, 2015) and this work will need to be expanded to these multi-functionality RepRap designs.

For electronics prototyping, in addition to the PCB milling demonstrated here, the platform could be used to print conductive, semiconducting (e.g. light emitting, photovoltiac, etc.) or magnetic inks, as a pick and place tool or for solder paste placement (masking). With further development, this tool could then fulfill much of the RepRap goal of becoming self-replicating to a larger extent than is currently possible. This tool can also be expanded to provide other forms of rapid prototyping. For example, for prototyping heat exchangers, this system could be adapted with a fiber laser to perform forward conduction welding to make expanded microchannel polymer heat exchangers (Denkenberger, et al., 2012).

Given the precise motion control capabilities of the platform, it can be useful for the development of open source scientific hardware (Pearce, 2014). For example, the platform could be developed as a low-cost microscope stage with heavy optical microscopes attached to the fixed tool mount or inexpensive USB digital microscopes attached to the tool effector. In addition, it could be adapted to serve as a low-cost micro-manipulation platform wherein users control motion while 
Gerald Anzalone, Bas Wijnen, Joshua M. Pearce, (2015) "Multi-material additive and subtractive prosumer digital fabrication with a free and open-source convertible delta RepRap 3-D printer", Rapid Prototyping Journal, 21(5), pp.506 - 519. doi:http://dx.doi.org/10.1108/RPJ-09-2014-0113

observing results in a magnified view. Open source labs could also benefit from utilizing the platform for profilometry, both contact and non-contact modes. The applications of such a tool to open-source labs is as varied and as expansive as all of the experimental sciences.

\section{Conclusions}

Open-source RepRap 3-D printers and their derivatives dominate the entry-level rapid prototyping landscape. The novel collection of extensions of the capabilities of RepRaps with the convertible delta RepRap investigated here expands affordable prototyping into new domains. The delta printer design performed a variety of manufacturing tasks and all of the tools were largely manufactured by the printer itself. The bill of materials, basic assembly and description are provided as free and open source hardware. This study has shown that as the tool remains stationary in the stage configuration, a tool having much greater mass can be fit above the build platform, expanding the catalog of tools and thus materials available for both additive and subtractive manufacturing on the delta platform. The convertible delta RepRap was then tested and profound to perform well with a variety of tools including: a thermoplastic extruder, a caulk/silicone extruder, a syringe pump extruder, a micro mill spindle and a tool holder used for vinyl cutting and plotting. The multi-functionality of this $<\$ 1000$ multi-material additive and subtractive fabrication tool was provided with cost savings of between $90-97 \%$ when compared to single-purpose tools with equivalent functionality.

\section{References}

Anzalone, G. C., Glover, A. G., \& Pearce, J. M. (2013). Open-source colorimeter. Sensors, 13(4), 5338-5346.

Anzalone, G., Zhang, C., Wijnen, B., Sanders, P., \& Pearce, J. (2013). A Low-Cost Open-Source Metal 3-D Printer. IEEE Access, 1, pp.803-810.

Betts, B. (2010). Bringing the factory home [personal fabrication technology]. Engineering \& Technology, 5(8), 56-58.

Baechler, C., DeVuono, M., \& Pearce, J. M. (2013). Distributed recycling of waste polymer into RepRap feedstock. Rapid Prototyping Journal, 19(2), 118-125.

Boldrin, M., \& Levine, D. (2002). The case against intellectual property. American Economic Review, 209-212.

Boldrin, M., \& Levine, D. K. (2008). Against intellectual monopoly (p. 7). Cambridge: Cambridge University Press.

Campbell, I., Bourell, D., \& Gibson, I. (2012). Additive manufacturing: rapid prototyping comes of age. Rapid Prototyping Journal, 18(4), 255-258.

Chu, A. C., Cozzi, G., \& Galli, S. (2012). Does intellectual monopoly stimulate or stifle innovation?. European Economic Review, 56(4), 727-746.

Cohen, D. L., Lipton, J. I., Cutler, M., Coulter, D., Vesco, A., \& Lipson, H. (2009). Hydrocolloid printing: a novel platform for customized food production. In the Solid Freeform Fabrication Symposium, 807-818.

Denkenberger, D. C., Brandemuehl, M. J., Pearce, J. M., \& Zhai, J. (2012). Expanded microchannel heat exchanger: design, fabrication, and preliminary experimental test. Proceedings of the Institution of Mechanical Engineers, Part A: Journal of Power and Energy, 226, 532-544.

Feeley, S. R., Wijnen, B., \& Pearce, J. M. (2014). Evaluation of Potential Fair Trade Standards for an Ethical 3-D Printing 
Gerald Anzalone, Bas Wijnen, Joshua M. Pearce, (2015) "Multi-material additive and subtractive prosumer digital fabrication with a free and open-source convertible delta RepRap 3-D printer", Rapid Prototyping Journal, 21(5), pp.506 - 519. doi:http://dx.doi.org/10.1108/RPJ-09-2014-0113

Filament. Journal of Sustainable Development, 7(5), 1-12.

Haselhuhn, A.S., Gooding, E.J. Glover, A.G., Anzalone, G.C., Wijnen, B., Sanders, P.G., Pearce, J.M. (2014). Substrate Release Mechanisms for Gas Metal Arc 3-D Aluminum Metal Printing. 3D Printing and Additive Manufacturing, 1(4): 204209.

Hoch, E., Tovar, G. E., \& Borchers, K. (2014). Bioprinting of artificial blood vessels: current approaches towards a demanding goal. European Journal of Cardio-Thoracic Surgery, ezu242.

Irwin, J. L., Pearce, J. M., Anzolone, G., \& Oppliger, D. E. (2014). The RepRap 3-D Printer Revolution in STEM Education. In 121st ASEE Annual Conference \& Exposition, Indianapolis, IN. Paper ID \#8696.

Jaffe, A. B., \& Lerner, J. (2011). Innovation and its discontents: How our broken patent system is endangering innovation and progress, and what to do about it. Princeton University Press

Jones, R., Haufe, P., Sells, E., Iravani, P., Olliver, V., Palmer, C., \& Bowyer, A. (2011). RepRap-the replicating rapid prototyper. Robotica, 29(01), 177-191.

Kentzer, J. , B. Koch, M. Thiim, R. W. Jones, E. Villumsen, (2011). An Open Source Hardware-based Mechatronics Project: The Replicating Rapid 3-D Printer, In the 4th International Conference On Mechatronics. IEEE. pp. 1-8, 2011.

Kreiger, M., \& Pearce, J. M. (2013). Environmental Life Cycle Analysis of Distributed Three-Dimensional Printing and Conventional Manufacturing of Polymer Products. ACS Sustainable Chemistry \& Engineering, 1(12), 1511-1519.

Kreiger, M. A., Mulder, M. L., Glover, A. G., \& Pearce, J. M. (2014). Life cycle analysis of distributed recycling of postconsumer high density polyethylene for 3-D printing filament. Journal of Cleaner Production, 70, 90-96.

Heller, M. A., \& Eisenberg, R. S. (1998). Can patents deter innovation? The anticommons in biomedical research. Science, 280(5364), 698-701.

Lakhani, K. R., \& Von Hippel, E. (2003). How open source software works:“free” user-to-user assistance. Research policy, 32(6), 923-943.

Lanzotti, A., Martorelli, M., \& Staiano, G. (2015). Understanding Process Parameter Effects of RepRap Open-Source Three-Dimensional Printers Through a Design of Experiments Approach. Journal of Manufacturing Science and Engineering, 137(1), 011017.

Moilanen, J. \& Vadén, T. (2012). Manufacturing in motion: first survey on the 3D printing community, Statistical Studies of Peer Production. http://surveys.peerproduction.net/2012/05/manufacturing-in-motion/

Molitch-Hou, M. (2013). Microfactory + FABtotum = The Rise of Hybrid $\infty$ Manufacturing. $3 D$ Printing Industry. available at:http://3dprintingindustry.com/2013/08/22/microfactory-fabtotum-the-rise-of-hybrid-manufacturing (accessed 11 September 2014).

Molitch-Hou, M. (2014). FABtotum Hybrid 3D Printing, Scanning, and Milling Machine Open for Pre-Orders. 3D Printing Industry. available at: http://3dprintingindustry.com/2014/09/03/fabtotum-hybrid-3d-printing-scanning-milling-machineopen-pre-orders (accessed 11 September 2014).

MOST. (2014), “Delta Build Overview:MOST” available at: http://www.appropedia.org/Delta Build Overview:MOST (accessed 11 September 2014). 
Gerald Anzalone, Bas Wijnen, Joshua M. Pearce, (2015) "Multi-material additive and subtractive prosumer digital fabrication with a free and open-source convertible delta RepRap 3-D printer", Rapid Prototyping Journal, 21(5), pp.506 - 519. doi:http://dx.doi.org/10.1108/RPJ-09-2014-0113

MTU-MOST. (2014), “SCAD libraries used in MOST OpenSCAD designs” available at: https://github.com/mtu-most/mostscad-libraries (accessed 11 September 2014).

Nettleton, D. S. (2013), “Orthotic Insoles (Supported).” available at: http://www.thingiverse.com/thing:47025 (accessed 11 September 2014).

Partridge, R., Conlisk, N., \& Davies, J. A. (2012). In-lab three-dimensional printing An inexpensive tool for experimentation. Organogenesis, 8(1).

Pearce, J. M. (2012). Building research equipment with free, open-source hardware. Science, 337(6100), 1303-1304.

Pearce, J. M. (2013). Open-source nanotechnology: Solutions to a modern intellectual property tragedy. Nano Today, 8(4), 339-341.

Pearce, J. M. (2014). Open-source lab: How to build your own hardware and reduce research costs. Elsevier.

Pearce, J. M. (2015). Quantifying the Value of Open Source Hard-ware Development. Modern Economy, 6(01), 1-11.

Pearce, J. M., Blair, C. M., Laciak, K. J., Andrews, R., Nosrat, A., \& Zelenika-Zovko, I. (2010). 3-D printing of open source appropriate technologies for self-directed sustainable development. Journal of Sustainable Development, 3(4), p17.

Rocholl, J. C. (2012). “Rostock” available at: http://reprap.org/wiki/Rostock (accessed 11 September 2014).

Sells, E., S. Bailard, Z. Smith, A. Bowyer, (2010). "Reprap: the replicating rapid prototype: maximizing curstomizability by breeding the means of production”, in F. T. Piller, M. M. Tseng (Eds.), Handbook of Research in Mass Customization and Personalization: Strategies and concepts. Vol. 1. World Scientific, pp. 568-580, 2010.

Stemp-Morlock, G. (2010). Personal fabrication. Communications of the ACM, 53(10), 14-15.

Takalo, T., \& Kanniainen, V. (2000). Do patents slow down technological progress?: Real options in research, patenting, and market introduction. International Journal of Industrial Organization, 18(7), 1105-1127.

Tymrak, B. M., Kreiger, M., \& Pearce, J. M. (2014). Mechanical properties of components fabricated with open-source 3-D printers under realistic environmental conditions. Materials \& Design, 58, 242-246.

Wijnen, B., Anzalone, G.C., Pearce, J.M., Free and open-source control software for 3-D motion and processing (to be published, 2015).

Wijnen, B., Hunt, E. J., Anzalone, G.C., Pearce, J.M. (2014). Open-source Syringe Pump Library, PLoS ONE 9(9): e107216.

Wittbrodt, B. T., A. G. Glover, J. Laureto, G. C. Anzalone, D. Oppliger, J. L. Irwin, and J. M. Pearce. "Life-cycle economic analysis of distributed manufacturing with open-source 3-D printers." Mechatronics 23, no. 6 (2013): 713-726.

ZeGo Robotics. (2014). "ZeGo Robotics” available at: http://www.zegorobotics.com/ (accessed 11 September 2014).

Zhang, C., Anzalone, N. C., Faria, R. P., \& Pearce, J. M. (2013). Open-source 3D-printable optics equipment. PloS One, 8(3), e59840. 